\title{
Formation of Electron-Root Radial Electric Field and its Effect on Thermal Transport in LHD High $T_{\mathrm{e}}$ Plasma
}

\author{
Seikichi MATSUOKA ${ }^{\text {a)}}$, Shinsuke SATAKE, Hiromi TAKAHASHI, Arimitsu WAKASA ${ }^{1)}$, \\ Masayuki YOKOYAMA, Takeshi IDO, Akihiro SHIMIZU, Takashi SHIMOZUMA, \\ Sadayoshi MURAKAMI ${ }^{1)}$ and LHD Experiment Group \\ National Institute for Fusion Science, Toki 509-5292, Japan \\ ${ }^{1)}$ Department of Nuclear Engineering, Kyoto University, Kyoto 606-8501, Japan
}

(Received 17 July 2012 / Accepted 5 March 2013)

\begin{abstract}
Neoclassical transport analyses have been performed for a high electron temperature LHD plasma with steep temperature gradient using a neoclassical transport simulation code, FORTEC-3D. It is shown that the large positive radial electric field is spontaneously formed at the core along with the increase in the electron temperature, while the neoclassical heat diffusivity remains almost unchanged. This indicates that the $1 / v$-type increase expected in the neoclassical transport in helical plasmas can be avoided by the spontaneous formation of the radial electric field. At the same time, it is found that the experimentally estimated heat diffusivity is significantly reduced. This suggests that the formation process of the transport barrier in the high electron temperature plasma can be caused by the spontaneous formation of the radial electric field.
\end{abstract}

(c) 2013 The Japan Society of Plasma Science and Nuclear Fusion Research

Keywords: radial electric field, finite orbit width effect, neoclassical transport, $\delta f$ Monte Carlo simulation

DOI: $10.1585 /$ pfr.8.1403039

\section{Introduction}

The formation of the electron internal transport barrier (eITB) observed in many helical devices $[1,2]$ is promising for the better confinement. High electron temperature $\left(T_{\mathrm{e}}\right)$ plasmas followed by the formation of the eITB are called CERC (Core Electron-Root Confinement) plasmas since they have a large positive (electron-root) radial electric field $\left(E_{\mathrm{r}}\right)$ at the plasma core. It has been observed that the $E_{\mathrm{r}}$ in a CERC plasma has a steep shear at the plasma core region where the steep $T_{\mathrm{e}}$ gradient of eITB forms. In the Large Helical Device (LHD), CERC plasmas are originally observed in the NBI-sustained plasmas with a superposition of electron cyclotron heating $(\mathrm{ECH})$ [3]. Recent experiments in LHD have shown that the high $T_{\mathrm{e}}$ plasmas can be observed with only ECH for relatively low density plasmas [4].

During the discharge of a CERC plasma, it has been observed in LHD that the $T_{\mathrm{e}}$ profile changes from flat into steep one as the ECH continues. In this process, a locally flat profile of $T_{\mathrm{e}}$ appears temporally, then the local flat region shrinks, and finally the $T_{\mathrm{e}}$ profile results in the steep eITB one. The resultant foot point of the eITB approaches the low order rational surface of $\iota / 2 \pi=1 / 2$. It has been pointed out that the position where the eITB formation occurs in CERC plasmas has a close relationship to either a low-order rational surface or a magnetic island [5]. It has been also reported that there is a threshold of the heating

author'se-mail: matsuoka@ rist.or.jp

a) Present address: Research Organization for Information Science and Technology, Kobe 650-0046, Japan. power to realize the electron-root $E_{\mathrm{r}}$, and it has a clear dependence on the magnetic axis position [6]. With these observations, however, the mechanism of the eITB formation has not been clarified. It is necessary to understand the mechanism how the eITB forms from the viewpoint of the transport. In general, the $E_{\mathrm{r}}$ and its shear strongly affect both the neoclassical and anomalous transport. Since CERC plasmas are characterized by the formation of the electron-root $E_{\mathrm{r}}$ at the core, it is important at first to investigate the $E_{\mathrm{r}}$ formation process and its relation to the transport reduction during the discharge. The $E_{\mathrm{r}}$ in helical plasmas can be determined by the ambipolar condition of the neoclassical transport, where the neoclassical particle fluxes of the electron and the ion balance. An accurate evaluation of the neoclassical transport and $E_{\mathrm{r}}$ is a key issue in the transport studies in helical plasmas.

In our recent work, it has been shown that the finite drift motion of electron orbits across the field lines, or the finite orbit width (FOW) effect on the neoclassical transport becomes important in high $T_{\mathrm{e}}$ helical plasmas due to the large deviation of the helically-trapped electrons [7]. A neoclassical transport code, FORTEC-3D, which was used in that study, has been developed with the aim to apply to such high $T_{\text {e }}$ plasmas. FORTEC-3D code has features below: (1) it involves the electron FOW effect in following the orbit of (marker) particles with the $\delta f$ Monte Carlo approach, (2) it can be applied to arbitrary magnetic field configurations such as tokamaks and helical/stellarator plasmas when the field is expressed in Boozer coordinates [8], 
(3) the collision operator for like-particles satisfies the elementary conservation laws for the particle number, the momentum, and the total energy, and (4) the steady-state ambipolar $E_{\mathrm{r}}$ is determined self-consistently by the ambipolar condition of Eq. (4) shown later. It is noted that the collision operator for unlike-species (here, the electron-ion collision) is represented only by the Lorentz operator, or the pitch angle scattering, since ion is assumed to be a stationary Maxwellian. This is due to the fact that any external momentum input for the ion does not exist, and thus the ion mean flow can be regarded negligible compared to the electron thermal speed. Although the momentum conservation in the unlike-particle collision is not satisfied for the collision operator, momentum transfer from electron to ion is negligible due to the large difference between the electron mass and ion one. With these features, FORTEC-3D code is aimed to evaluate the neoclassical transport and the ambipolar $E_{\mathrm{r}}$ more accurately in high temperature plasmas.

In this paper, FORTEC-3D code is applied to a LHD CERC plasma to clarify characteristics in the formation of $E_{\mathrm{r}}$ and its effect on the electron thermal transport. we show that the ambipolar $E_{\mathrm{r}}$ spontaneously grows to have a large positive electron root with the steep shear as the $T_{\mathrm{e}}$ gets high and the eITB formation proceeds. On the other hand, the electron neoclassical heat diffusivities remain almost constant due to the electron-root $E_{\mathrm{r}}$. It is kept lower level than expected from the so-called $1 / v$ scaling in which the heat diffusivity increases in proportion to $T_{\mathrm{e}}^{7 / 2}$ in helical plasmas. It indicates that the spontaneous formation of the electron-root $E_{\mathrm{r}}$ compensates the increase in the electron thermal transport in the CERC plasma. The experimental heat diffusivities estimated from the input power of the $\mathrm{ECH}$ is compared to the neoclassical ones.

The rest of this paper is organized as follows. In Sec. 2, the brief description of a numerical neoclassical transport code, FORTEC-3D, and the ambipolar condition for the $E_{\mathrm{r}}$ is presented. The importance of the electron FOW effect on the electron neoclassical transport is also described in this section. Section 3 gives numerical results of neoclassical transport analyses for a LHD CERC plasma. Finally, a summary and a discussion are given in Sec. 4.

\section{Neoclassical Transport Code with Finite Orbit Width Effect, FORTEC-3D}

FORTEC-3D solves the drift kinetic equation based on the two-weight $\delta f$ Monte Carlo method [9-11]. It determines the neoclassical particle and energy fluxes by following the motion of a large number of marker particles in an arbitrary magnetic field. In the two-weight $\delta f$ Monte Carlo method, the distribution function $f_{a}$ is decomposed into two parts as $f_{a}=f_{a, 0}+\delta f_{a}$, where $a=\mathrm{e}$, i denotes a plasma species and $f_{a, 0}=f_{a, \mathrm{M}}$ is the Maxwellian distribution function. The following drift kinetic equation for the $\delta f_{a}$ part of the distribution should be solved using simulation markers,

$$
\begin{aligned}
\frac{D \delta f_{a}}{D t} & \equiv\left[\frac{\partial}{\partial t}+\left(\boldsymbol{v}_{\|}+\boldsymbol{v}_{\mathrm{d}}\right) \cdot \nabla+\dot{K} \frac{\partial}{\partial K}-C_{\mathrm{TP}}\right] \delta f_{a} \\
& =\left[-\left(\boldsymbol{v}_{\|}+\boldsymbol{v}_{\mathrm{d}}\right) \cdot \nabla+C_{\mathrm{FP}}\right] f_{a, \mathrm{M}},
\end{aligned}
$$

where $\boldsymbol{v}_{\|}$and $\boldsymbol{v}_{\mathrm{d}}$ are the parallel and drift velocity of particles, and $C_{\mathrm{TP}}$ and $C_{\mathrm{FP}}$ are the test particle and field particle collision operators, respectively. In FORTEC-3D, the electron-electron collision operator satisfies the conservation laws for the particle number, momentum, and the total energy (see Ref. [11]), while the electron-ion one only involves the pitch angle scattering. The assumption that the ion distribution function is a stationary Maxwellian and its mean flow $U_{\mathrm{i}}$ is negligibly small compared with the electron thermal speed, $v_{\text {th,e }} \gg v_{\text {th,i }} \simeq U_{\mathrm{i}} \simeq 0$, enables FORTEC-3D to use such simplified collision operator for the electron-ion collision, where $v_{\text {th,e }}$ and $v_{\text {th,i }}$ are the electron and ion thermal velocities, respectively. The five-dimensional phase space variables, $(\boldsymbol{R}, K, \mu)$, are used here, where $\boldsymbol{R}$ represents the position vector in Boozer coordinates, $K$ is the kinetic energy, and $\mu$ is the magnetic moment. The $f_{a, 0}=f_{a, \mathrm{M}}$ does not have the mean velocity here, and thus it does not contribute to any radial transport. Hence, the neoclassical transport in the radial direction is only obtained by moments of the $\delta f$ in velocity spaces. The neoclassical radial particle and energy fluxes $\Gamma_{a}$ and $Q_{a}$ are given as follows:

$$
\begin{aligned}
\Gamma_{a} & =\int v_{\mathrm{r}} \delta f_{a} \mathrm{~d}^{3} v, \\
Q_{a} & =\int \frac{1}{2} m_{a} v^{2} v_{\mathrm{r}} \delta f_{a} \mathrm{~d}^{3} v,
\end{aligned}
$$

where $v$ and $v_{\mathrm{r}}$ are the velocity and its radial component, and $m_{a}$ is the mass of $a$-th species.

In referring to the FOW effect in this paper, we mean the whole effect caused by neglecting the particle radial drift. This comes from the convention of the local neoclassical transport adopted in many numerical neoclassical transport codes, such as DKES [12]. In the local codes, not only the radial drift but also the $\nabla B$ and curvature drifts in poloidal and toroidal directions included in the drift kinetic equation are neglected as a consequence of the assumption of $\left|v_{\boldsymbol{E} \times \boldsymbol{B}}\right| \gg\left|v_{B}\right|$, where $v_{\boldsymbol{E} \times \boldsymbol{B}}$ is the $\boldsymbol{E} \times \boldsymbol{B}$ drifts and $v_{B}$ represents the drift arising from the $\nabla B$ and the curvature. It is noted that the incompressible $v_{\boldsymbol{E} \times \boldsymbol{B}}$ is also usually assumed there. These assumptions are not necessary for FORTEC-3D since all the drifts above are included in FORTEC-3D. The FOW effect on the neoclassical transport arises through the collisionless detrapping of the helically-trapped particles and the poloidal resonance. The former one makes the $1 / v$ neoclassical transport flux smaller due to the less fraction of the helically-trapped particles as a consequence of the collisionless detrapping of trapped particles. With the latter effect, the neoclassical transport flux of purely $1 / v$-behavior appears with a small 
but finite $E_{\mathrm{r}}$. More detailed discussion on the electron FOW effect can be seen in Ref. [7].

In this paper, only the electron neoclassical transport is evaluated by FORTEC-3D with its FOW taken into account. The reason for this is twofold: one is that the electron FOW effect makes a significant influence on the electron neoclassical transport when $T_{\mathrm{e}}$ is high due to helicallytrapped particles in a three-dimensional magnetic field [7] and the other is that the ion temperature is in general low in CERC plasmas.

The time evolution of $E_{\mathrm{r}}$ can be described by the following equation,

$$
\epsilon_{\perp} \epsilon_{0} \frac{\partial E_{\mathrm{r}}}{\partial t}=-e\left\{\Gamma_{\mathrm{e}}\left(E_{\mathrm{r}}, U_{\mathrm{e}, \|}\right)-\Gamma_{\mathrm{i}}\left(E_{\mathrm{r}}, U_{\mathrm{i}, \|}\right)\right\},
$$

where $U_{a, \|}$ is the parallel mean flow of species $a, \epsilon_{0}$ represents the electric permittivity in the vacuum, and $\epsilon_{\perp}$ denotes the effect of the classical polarization current defined by

$$
\epsilon_{\perp} \equiv\left\langle|\nabla \rho|^{2}\right\rangle+\left\langle\frac{v_{\mathrm{A}}^{2}}{c^{2}}|\nabla \rho|^{2}\right\rangle,
$$

where $v_{\mathrm{A}}$ is the Alfvèn velocity and $c$ is the speed of light. The radial electric field is determined in FORTEC-3D by imposing the ambipolar condition for the neoclassical particle fluxes of electrons and ions. It is realized when the left hand side of Eq. (4) vanishes: $\Gamma_{\mathrm{e}}$ and $\Gamma_{\mathrm{i}}$ balance and $E_{\mathrm{r}}$ reaches a steady state. Hence, one needs to evaluate the ion neoclassical particle flux in addition to the electron one although only the latter is evaluated by FORTEC-3D in this paper. Since it requires the large amount of computational resources to simultaneously calculate both the ion and the electron neoclassical transport fluxes by FORTEC3D, a database made by DGN/LHD [13], which is a numerical neoclassical transport code without the FOW effect, is adopted for the ion particle flux as is done in the reference [14]. The ion particle flux $\Gamma_{\mathrm{i}}$ is referred to from the corresponding radial position $\rho$ and the radial electric field $E_{\mathrm{r}}$. This enables ones to evaluate $\Gamma_{\mathrm{i}}$ at each time step without much computational burden. Therefore, the $E_{\mathrm{r}}$ is determined by FORTEC-3D to meet the ambipolar condition of $\Gamma_{\mathrm{e}}^{\mathrm{F} 3 \mathrm{D}}\left(E_{\mathrm{r}}^{\mathrm{F} 3 \mathrm{D}}\right)-\Gamma_{\mathrm{i}}^{\mathrm{DGN}}\left(E_{\mathrm{r}}^{\mathrm{F} 3 \mathrm{D}}\right)=0$, where the superscripts of F3D and DGN refer to the value obtained by FORTEC-3D and DGN/LHD, respectively. It is noted that the $E_{\mathrm{r}}$ can also be determined by using only DGN/LHD as $\Gamma_{\mathrm{e}}^{\mathrm{DGN}}\left(E_{\mathrm{r}}^{\mathrm{DGN}}\right)-\Gamma_{\mathrm{i}}^{\mathrm{DGN}}\left(E_{\mathrm{r}}^{\mathrm{DGN}}\right)=0$, and the $E_{\mathrm{r}}^{\mathrm{DGN}}$ such obtained by DGN/LHD is also shown in the next section for the reference purpose. Finally, it is noted that the averages over certain time steps for resultant values of FORTEC$3 \mathrm{D}$ such as $E_{\mathrm{r}}$, the heat diffusivity, etc., are shown in the remaining part of the paper to remove an inevitable numerical noise of the Monte Carlo method as much as possible.

\section{The Radial Electric Field Forma- tion in CERC Plasma}

The FORTEC-3D code is applied to a CERC plasma observed in the LHD experiment of \# 103619. The heating scenario of \# 103619 is shown in Fig. 1. The ECH with two gyrotrons of $77 \mathrm{GHz}$ has been operated and the total injection power of the ECH was $1.34 \mathrm{MW}$ for the discharge. The momentum input by ECH in the parallel direction is not taken into account in simulations here since that is small in this discharge. The time evolution of the electron temperature at the magnetic axis of $R=3.53 \mathrm{~m}$ and the line averaged density are also shown in the figure, where $R$ is the major radius. The magnetic field strength at the axis is $B_{\mathrm{ax}}=2.705 \mathrm{~T}$. It is noted that the $\beta$ value is very low for all the timing of this discharge $(\beta<0.02 \%)$ due to the low density of the plasma. In FORTEC-3D, the effect of the heating power on the neoclassical transport and the $E_{\mathrm{r}}$ formation is not considered. This is justified by the fact that the nonambipolar flux of energetic particles driven by ECH does not contribute so much to the ambipolar condition, and thus the resultant ambipolar $E_{\mathrm{r}}$ for a similar plasma density as the CERC plasma considered here [1].

The electron temperature of the LHD \# 103619 discharge observed by the Thomson scattering are shown in Fig. 2 (a). As shown in the figure, $T_{\mathrm{e}}$ at $t=0.8 \mathrm{~s}$ (denoted by flat) shows a flat profile over the core region of approximately at $\rho<0.4$, a locally flat one at $t=0.9 \mathrm{~s}$ (local flat), and an eITB one at $t=1.1 \mathrm{~s}(e I T B)$. It can be seen that the local flat temperature profile gradually vanishes as the ECH continues as mentioned in Sec. 1. The ion temperature is not available for the discharge, thus the parabolic profile for $T_{\mathrm{i}}$ is assumed with $T_{\mathrm{i}}=0.4 \mathrm{keV}$ at the magnetic axis for all the timing of the discharge as shown in the Fig. 2 (a). The use of the local neoclassical transport code, DGN/LHD, for $\Gamma_{\mathrm{i}}$ is justified due to the low $T_{\mathrm{i}}$ of the

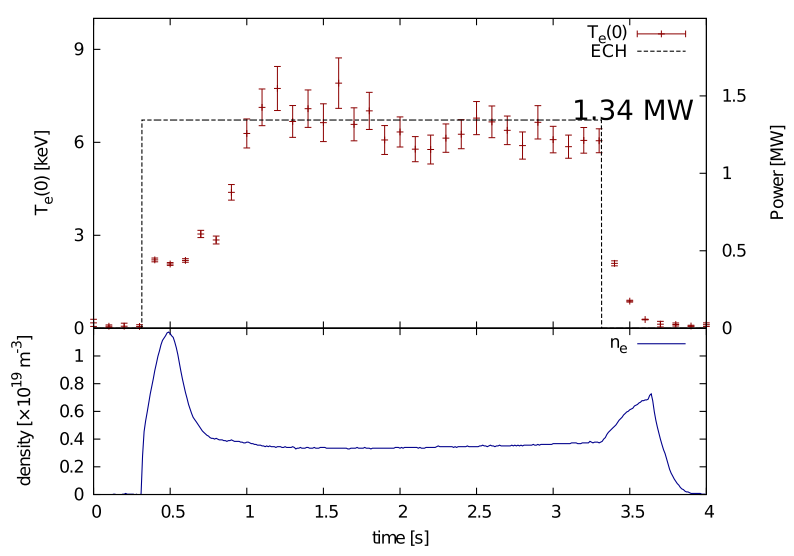

Fig. 1 The time sequence of the ECH of the LHD \# 103619 discharge. The total power of $1.34 \mathrm{MW}$ with two $77 \mathrm{GHz}$ gyrotrons was injected for the plasma. The electron temperature at $R_{\mathrm{ax}}=3.53 \mathrm{~m}$ and line-averaged density are also shown. 
(a)

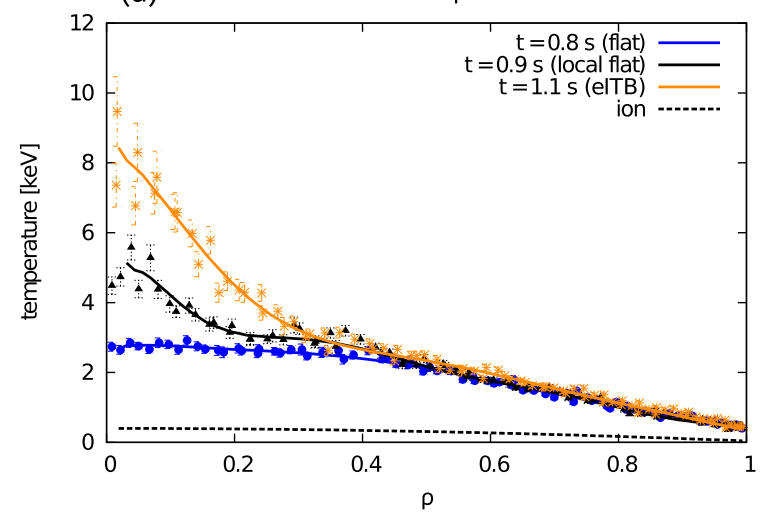

(b)

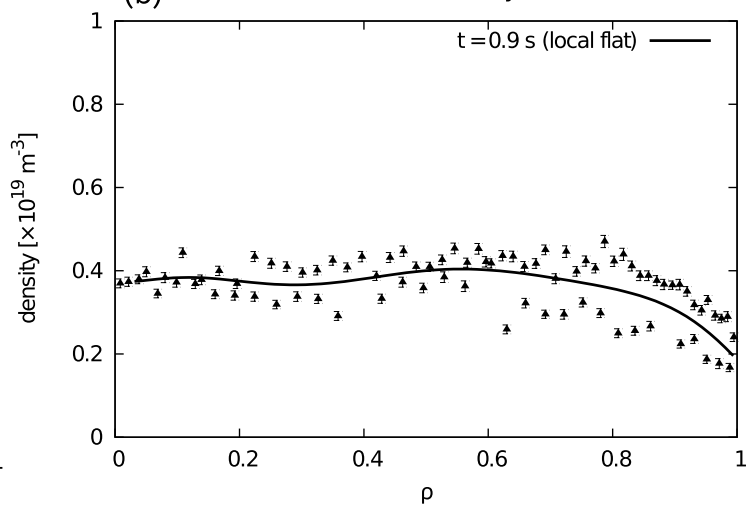

Fig. 2 (a) The temperature profiles of the LHD CERC plasma (\# 103619) for several timing. The ion temperature, which is assumed as the same value for all timing due to the lack of the observation, is indicated by a dashed line. (b) The density profile of the discharge. Since the density remains almost constant after $t \simeq 0.8 \mathrm{~s}$, only the value at $t=0.9 \mathrm{~s}$ is indicated for simplicity.

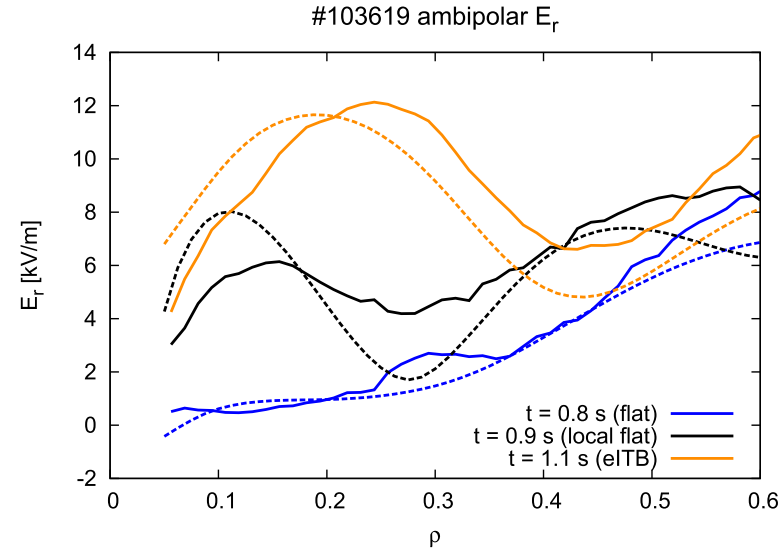

Fig. 3 The ambipolar radial electric field profiles for the LHD CERC plasma obtained by FORTEC-3D. Each $E_{\mathrm{r}}$ profile shows a value at the steady state. The ambipolar $E_{\mathrm{r}}$ obtained by DGN/LHD are also plotted in the figure (dashed lines).

CERC plasma. The plasma density of the discharge is flat and low $\left(n_{\mathrm{e}} \simeq 0.4 \times 10^{19} \mathrm{~m}^{-3}\right)$ as shown in Fig. $2(\mathrm{~b})$. The plasma density remains almost constant after $t \simeq 0$.8s (see also Fig. 1), thus only the value at $t=0.9 \mathrm{~s}$ is indicated for simplicity. It is noted that FORTEC-3D calculates the neoclassical transport for fitted profiles of $T_{\mathrm{e}}$ and $n_{\mathrm{e}}$ shown in Figs. 2 (a) and (b) and equilibrium magnetic fields obtained by VMEC code [15].

The FORTEC-3D simulations presented in this paper have used $2 \times 10^{7}$ markers for the lower $T_{\mathrm{e}}$ cases $(t=0.9$ and $1.0 \mathrm{~s})$ and $4 \times 10^{7}$ for high $T_{\mathrm{e}}$ case $(t=1.1 \mathrm{~s})$. Since the larger thermal velocity of the high $T_{\mathrm{e}}$ plasmas can degrade the accuracy of the simulation results, twice as much markers are used in the latter case. For $t=1.1 \mathrm{~s}$ case, we have confirmed the convergence of simulation results by varying the number of markers $N_{\mathrm{m}}=1 \times 10^{7}, 2 \times 10^{7}$ and $4 \times 10^{7}$. It has been confirmed that the ambipolar $E_{\mathrm{r}}$ is al- most unaffected by $N_{\mathrm{m}}$, and $Q_{\mathrm{e}}$ changes $20-30 \%$ at most in the core region and is unaffected at $\rho>0.3$.

The radial electric field obtained by FORTEC-3D is shown in Fig. 3. In this figure, $E_{\mathrm{r}}^{\mathrm{F} 3 \mathrm{D}}$ at $t=0.8$ (denoted by flat), 0.9 (local flat), and $1.1 \mathrm{~s}$ (eITB) are shown, respectively. Dashed lines in the figure represent results obtained by DGN/LHD, that is, $E_{\mathrm{r}}^{\mathrm{DGN}}$. As indicated in the figure, the ambipolar electron-root $E_{\mathrm{r}}$ with the steep shear is spontaneously formed along with the eITB formation from $t=0.8$ to $1.1 \mathrm{~s}$ at the core region. In the low $T_{\mathrm{e}}$ plasma at $t=0.8 \mathrm{~s}$, the ambipolar $E_{\mathrm{r}}$ of FORTEC-3D and DGN/LHD almost agree with each other. This is because the FOW effect in the low $T_{\mathrm{e}}$ plasma is small so that the electron particle fluxes of both codes are almost the same. On the other hand, the difference between FORTEC-3D and DGN/LHD becomes larger at $t=1.1 \mathrm{~s}$ when $T_{\mathrm{e}}$ increases especially at around $\rho=0.14$ where the steep $T_{\mathrm{e}}$ gradient forms, and it results in approximately $20 \%$ at the most there.

As described in Sec. 2, the radial electric field is determined by the electron and ion particle fluxes to satisfy the ambipolar condition of $\Gamma_{\mathrm{e}}\left(E_{\mathrm{r}}, U_{\mathrm{e}, \|}\right)=\Gamma_{\mathrm{i}}\left(E_{\mathrm{r}}, U_{\mathrm{i}, \|}\right)$. Note here that $\Gamma_{\mathrm{e}}$ from FORTEC-3D depends implicitly on the electron parallel flow, too. The electron parallel flow, defined as $n_{\mathrm{e}} U_{\mathrm{e}, \|}=\int v_{\|} \delta f \mathrm{~d}^{3} v$ in FORTEC-3D, can be seen as one that evolves according to the $m_{\mathrm{e}} v_{\|}$-moment of the drift kinetic equation (1):

$$
m_{\mathrm{e}} n_{\mathrm{e}} \frac{\partial U_{\mathrm{e}, \|}}{\partial t}=-\left\langle\boldsymbol{B} \cdot \nabla \cdot \boldsymbol{\Pi}_{\mathrm{e}}\right\rangle+\left\langle\boldsymbol{B} \cdot \boldsymbol{F}_{\mathrm{e}}\right\rangle,
$$

where $\boldsymbol{\Pi}_{\mathrm{e}}$ is the electron viscosity tensor, and $\boldsymbol{F}_{\mathrm{e}}$ is the friction force between electron and ion, respectively. $\Gamma_{\mathrm{i}}$ in the ambipolar condition is given as a function of the radial position $\rho$ and $E_{\mathrm{r}}$ at each time step in FORTEC-3D from a $\Gamma_{\mathrm{i}}$-database made by DGN/LHD code, which determines the steady-state $\Gamma_{\mathrm{i}}$ without including the ion-electron collision operator due to the small mass ratio $m_{\mathrm{e}} \ll m_{\mathrm{i}}$ (for more detailed description of DGN/LHD, see Ref. [13] and references therein). On the other hand, in the paral- 
lel momentum balance for ions, which has a similar form as Eq. (6), the ion-electron friction can be neglected again due to the small mass ratio. Complete momentum balance between ions and electrons is required only to explain the intrinsic ambipolarity in axisymmetric tokamaks. In LHD cases, ion-electron friction term has little effect in determining both ion parallel flow and the ambipolar condition because of finite neoclassical toroidal viscosity $\left\langle\boldsymbol{B}_{\mathrm{t}} \cdot \nabla \cdot \boldsymbol{\Pi}_{\mathrm{i}}\right\rangle$ in helical plasmas [16]. Moreover, since we analyze here ECH-heated plasmas, there is no explicit momentum source for ion and the assumption $v_{\text {th,e }} \gg U_{\mathrm{i}} \simeq 0$ in our model is valid. Therefore, although the $\Gamma_{\mathrm{i}}$-database from DGN/LHD code neglects the effect of ion-electron friction, and as a result, our simulation model of FORTEC$3 \mathrm{D}$ does not treat the momentum transfer from electron to ion, the use of the $\Gamma_{\mathrm{i}}$-database is justified to determine the ambipolar $E_{\mathrm{r}}$ in our simulation model for LHD under the $m_{\mathrm{e}} / m_{\mathrm{i}} \rightarrow 0$ limit approximation. The electron parallel flow and the $E_{\mathrm{r}}$ such obtained in FORTEC-3D simultaneously satisfy both the ambipolar condition and the parallel momentum balance Eq. (6).

The $E_{\mathrm{r}}$ and the electron mean flow also satisfy the radial force balance relation. The radial force balance relation is obtained from the momentum balance equation and is expressed as follows:

$$
E_{\psi}+\frac{p_{\mathrm{e}}^{\prime}}{e n_{\mathrm{e}}}+U^{\theta}=\frac{\iota}{2 \pi} U^{\zeta},
$$

where the radial electric field is expressed in $\psi$-coordinate instead of $r, p_{\mathrm{e}}^{\prime}$ is the pressure gradient, and $U^{\theta}=\boldsymbol{U} \cdot \nabla \theta$ and $U^{\zeta}=\boldsymbol{U} \cdot \nabla \zeta$ are contravariant components in theta- and zeta-directions of the electron mean flow, $n \boldsymbol{U}=\int \boldsymbol{v} \delta f \mathrm{~d}^{3} v$. In the present cases, it is found that $U / v_{\mathrm{th}, \mathrm{e}} \sim O\left(10^{-4}\right)$ and $U^{\zeta} / U^{\theta}<O\left(10^{-1}\right)$. This makes the $U^{\zeta}$ term in the right hand side of Eq. (7) negligible, leading to the $E_{\psi}+p_{\mathrm{e}}^{\prime} / e n_{\mathrm{e}}+$ $U^{\theta} \simeq 0$. To see this more in detail, the radial force balance for $t=1.1 \mathrm{~s}$ case is shown in Fig. 4. As clearly seen in the figure, the sum of the left hand side (black line) is almost zero over the wide region of the plasma, and the $U^{\zeta}$ term contributes little to the force balance (see dashed pink and orange lines in the figure).

The energy flux, $Q_{\mathrm{e}}$, and the heat diffusivity, $\chi_{\mathrm{e}}^{\mathrm{NC}}$ of those plasmas obtained by FORTEC-3D are shown in Figs. 5 (a) and (b). It is noted that the energy flux represents the total heat flux, that is, the sum of the convective and conductive heat fluxes. The energy fluxes calculated by DGN/LHD are also shown by dashed lines in Fig. 5 (a). The difference of the energy flux between FORTEC-3D and DGN/LHD at $t=0.9$ and $1.1 \mathrm{~s}$ are approximately $20-30 \%$ at $\rho \simeq 0.14$, which is slightly larger than that of $E_{\mathrm{r}}$. This is explained as follows. Particles used in FORTEC-3D have various energies, while DGN/LHD uses the mono-energetic particle to evaluate the neoclassical transport. Since the energy flux is evaluated by $v^{2}$ moment of the particle distribution function $\delta f$, the existence of such energy-distributed particles in FORTEC-3D

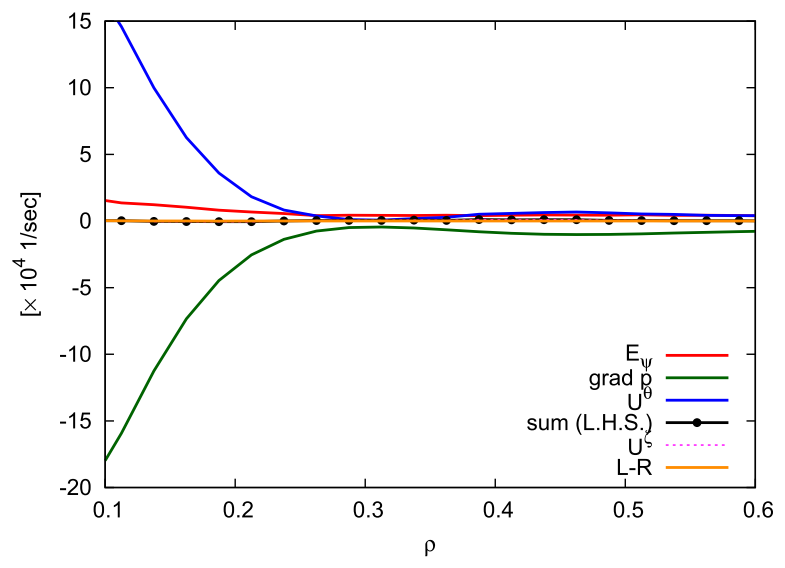

Fig. 4 Force balance relation in LHD plasma of \#103619 at $t=$ $1.1 \mathrm{~s}$. In the figure, red line represents the $E_{\psi}$ term in left hand side of Eq. (7), green the pressure gradient, blue $U^{\theta}$ and black with point the sum of them. Dashed pink line represents the $U^{\zeta}$ term in the right hand side of the same equation, and orange is the difference between black and orange lines. It is noted that the terms shown here are averaged over the magnetic surface.

more affects the energy flux rather than the particle flux and the ambipolar $E_{\mathrm{r}}$, where the former is the constraint of the ambipolar condition of $\Gamma_{\mathrm{e}}=\Gamma_{\mathrm{i}}$. It is noted that the ion particle flux, $\Gamma_{\mathrm{i}}$, is evaluated by DGN/LHD. The difference in the energy flux arises from this fact in addition to the difference in the ambipolar $E_{\mathrm{r}}$ seen in Fig. 3. The neoclassical heat diffusivities are shown by solid lines in Fig. 5 (b). It is noted that $\chi_{\mathrm{e}}^{\mathrm{NC}}$ shown in the figure represents effective values with respect to this energy flux, and are defined as $\chi_{\mathrm{e}}^{\mathrm{NC}} \equiv Q_{\mathrm{e}} /\left(-n_{\mathrm{e}} \nabla T_{\mathrm{e}}\right)$. Although the energy flux increases due to the increase in $T_{\mathrm{e}}$, the neoclassical heat diffusivity at the core rather remains low level even when $T_{\mathrm{e}}$ becomes high at $t=0.9$ and $1.1 \mathrm{~s}$. This is due to the formation of the large electron-root $E_{\mathrm{r}}$ during the eITB formation shown in Fig. 3. With the large electron-root $E_{\mathrm{r}}$ in the CERC plasma, the neoclassical heat diffusivity does not show the so-called $1 / v$ scaling which is expected to be large in high temperature plasmas if $E_{\mathrm{r}}=0$. In other words, the spontaneously growing electron-root $E_{\mathrm{r}}$ compensates the $1 / v$ increase in $\chi_{\mathrm{e}}^{\mathrm{NC}}$ when the eITB formation occurs in the CERC plasma. The neoclassical heat diffusivities are compared to experimental values, which are estimated from the power deposition profile of the $\mathrm{ECH}$, in Fig. 5 (b). The experimental heat diffusivities $\chi_{\mathrm{e}}^{\mathrm{EXP}}$ at the core are drastically reduced by the order of magnitude of $10^{1}$ or more from $t=0.8 \mathrm{~s}$ to $1.1 \mathrm{~s}$. Since the neoclassical part of the transport, $\chi_{\mathrm{e}}^{\mathrm{NC}}$ remains unchanged during the discharge, the reduction of the experimental heat diffusivity can be mainly attributed to the reduction of the anomalous, or the turbulent transport.

To explore the characteristics of the neoclassical transport further, dependence on the plasma collisionality is 

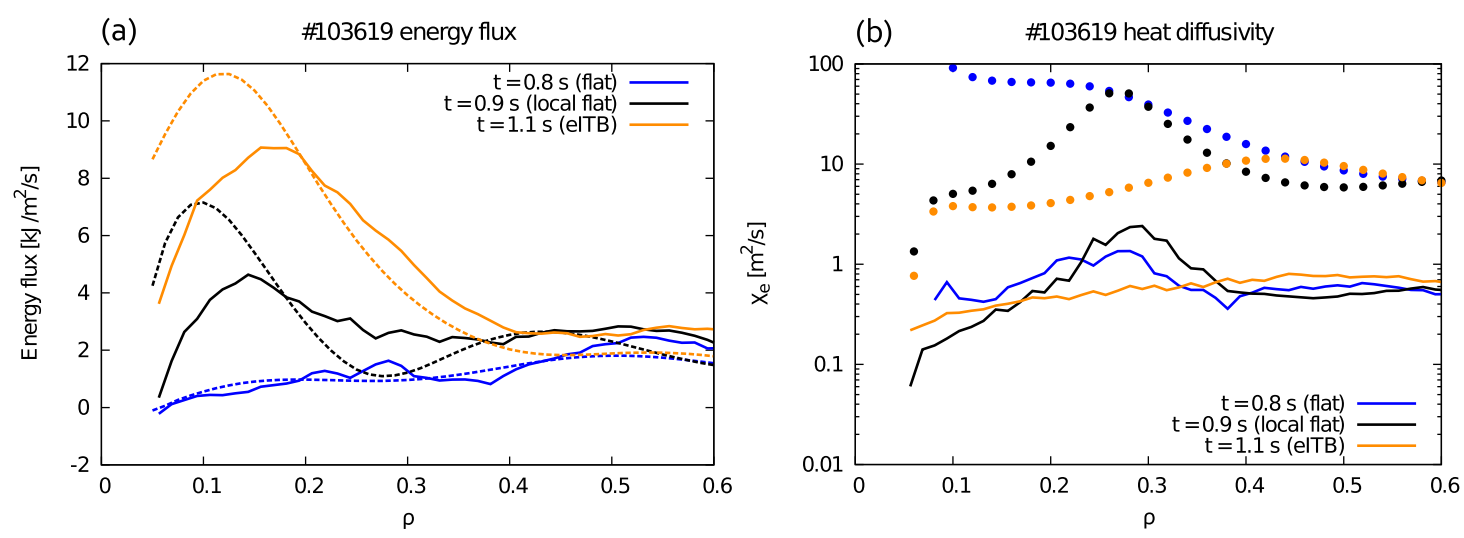

Fig. 5 (a) Radial profiles of the energy flux evaluated by FORTEC-3D (solid lines) and DGN/LHD (dashed lines) at $t=0.8,0.9$ and $1.1 \mathrm{~s}$ of the LHD \# 103619 discharge. (b) Radial profiles of the neoclassical heat diffusivities, $\chi_{\mathrm{e}}^{\mathrm{NC}}$ (solid lines) and experimental ones, $\chi_{\mathrm{e}}^{\mathrm{EXP}}$ (circles). The former ones are obtained by FORTEC-3D and the latter are estimated from the power deposition of the ECH.
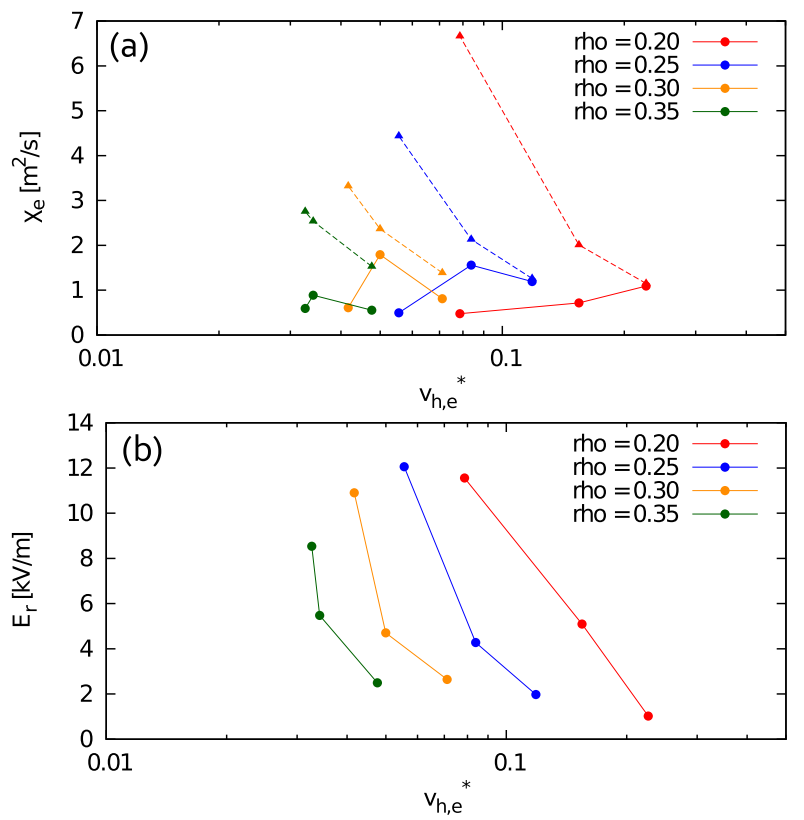

Fig. 6 (a) Neoclassical heat diffusivities evaluated by FORTEC3D (solid lines with symbols) vs. the normalized collisionality, $v_{\mathrm{h}, \mathrm{e}}^{*}$ at several radial positions. In (a), dashed lines with symbols corresponds to values with artificially setting $E_{\mathrm{r}}=0$. (b) The radial electric field evaluated by FORTEC-3D (solid lines with symbols) vs. the normalized collisionalities at the same positions as those in (a).

investigated. The heat diffusivities and the ambipolar $E_{\mathrm{r}}$ at several radial positions of $\rho=0.20,0.25,0.30$ and 0.35 are plotted with the collisionality in Figs. 6 (a) and (b). The normalized collisionality is defined as $v_{\mathrm{h}, \mathrm{e}}^{*}=$ $v /\left[\epsilon_{\mathrm{h}}^{3 / 2}\left(v_{\mathrm{th}, \mathrm{e}} / q R\right)\right]$, where $v$ denotes the collision frequency, and $\epsilon_{\mathrm{h}}$ and $q$ are the helicity of the LHD magnetic configuration and the safety factor, respectively. For comparisons with the $1 / v$-type dependence of the heat diffusivity, $\chi_{\mathrm{e}}^{\mathrm{NC}}$ evaluated from DGN/LHD by setting $E_{\mathrm{r}}=0$ are also plot-

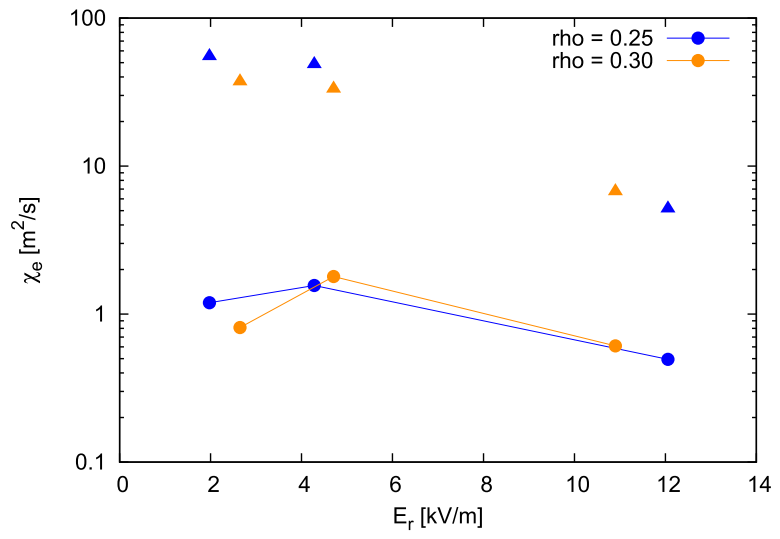

Fig. 7 The Heat diffusivities vs. the radial electric field. Solid lines represent the neoclassical ones obtained by FORTEC-3D, and triangles represent experimental estimations which are the same as those in Fig. 5 (b).

ted by dashed lines in the Fig. 6(a). It is clearly shown in the figure that $\chi_{\mathrm{e}}^{\mathrm{NC}}$ clearly decreases due to the larger value of $E_{\mathrm{r}}$ as the collisionality ( $T_{\mathrm{e}}$ ) decreases (increases), see solid lines in Figs. 6(a) and (b). It can be concluded that the $1 / v$ dependence of the heat diffusivity is clearly avoided at all the radial positions with the formation of the electron-root $E_{\mathrm{r}}$ when the collisionality becomes low. This shows that the neoclassical heat diffisivity results in either $\sqrt{v}$ - or $v$-regime with the ambipolar $E_{\mathrm{r}}$ instead of $1 / v$-regime. Figure 7 shows the dependence of both $\chi_{\mathrm{e}}^{\mathrm{NC}}$ and $\chi_{\mathrm{e}}^{\mathrm{EXP}}$ on the ambipolar $E_{\mathrm{r}}$ realized at $\rho=0.25$ and 0.30 . One can see the clear reduction of the experimental heat diffusivity in the figure, while the neoclassical heat diffusivities at both positions almost remain constants during the discharge in spite of the increase in $T_{\mathrm{e}}$ there.

\section{Summary and Discussion}

To investigate the radial electric field and its effect on 
the electron neoclassical thermal transport in a LHD CERC plasma, we have performed the neoclassical transport analyses using FORTEC-3D, which evaluates the neoclassical transport and the ambipolar $E_{\mathrm{r}}$ including the electron FOW effect. The CERC plasma is characterized by the high electron temperature $\left(T_{\mathrm{e}}>8 \mathrm{keV}\right.$ at the core) with the steep gradient of the eITB and the large positive $E_{\mathrm{r}}$, or the electron root with its steep shear. As pointed out in Ref. [7], the high $T_{\mathrm{e}}$ in a CERC plasma requires the electron FOW effect included in neoclassical transport analyses, and FORTEC-3D is appropriate tool for this purpose. It should be emphasized that this is the first attempt of the neoclassical transport analysis with the electron FOW for such a high $T_{\mathrm{e}}$ CERC plasma in LHD.

The radial electric field shows the spontaneous formation resulting in the large electron-root value with the eITB formation in the CERC plasma. The resultant $\chi_{\mathrm{e}}^{\mathrm{NC}}$ remains unchanged and kept lower values over the entire region inside the eITB foot point although the neoclassical thermal transport itself increases along with the increase in $T_{\mathrm{e}}$. This indicates that the spontaneous formation of the large electron-root $E_{\mathrm{r}}$ compensates the $1 / v$ degrading of the neoclassical transport. At the same time, the experimental heat diffusivity shows a clear reduction by the order of 10 or so during the eITB formation although it still remains much larger than the neoclassical transport level.

The ETG turbulence is attributed to the remaining transport since the steep $T_{\mathrm{e}}$ gradient exists in the CERC plasma. The effect of the $\boldsymbol{E} \times \boldsymbol{B}$ velocity shear on the growth of the turbulence plays a significant role for the transport reduction, see a review paper by Burrell [17] and references therein. Also it has been shown experimentally that the suppression of the turbulence-driven transport by the $\boldsymbol{E} \times \boldsymbol{B}$ velocity shear results in the transport barrier in helical devices [18]. As described above, the considerable reduction of the transport accomplished in the CERC plasma is realized with the spontaneous formation of the electron-root $E_{\mathrm{r}}$ with the large shear. Therefore, it can be expected that either the neoclassical ambipolar $E_{\mathrm{r}}$ and/or its shear results in the improved confinement of the CERC plasma. It is required to investigate either of these mechanisms more contribute to the reduction of the turbulent transport in the CERC plasma: the large electron-root $E_{\mathrm{r}}$ itself or the steep $E_{\mathrm{r}}$ shear at the eITB region. The linear growth rate of the ETG turbulence obtained by a gyrokinetic transport code, GKV-X [19], will be compared to the $E_{\mathrm{r}}$ shearing rate to discuss the turbulent transport in the CERC plasma in a forthcoming paper.

\section{Acknowledgments}

The authors would like to acknowledge Prof. Hideo Sugama (NIFS) for fruitful discussions on the transport physics in helical plasmas. This work is supported in part by JSPS Grant-in-Aid for Young Scientists (B), No. 23760810 and in part by the NIFS Collaborative Research Programs, NIFS12KNST038.

[1] K. Ida et al., Phys. Rev. Lett. 91, 085003 (2003).

[2] M. Yokoyama et al., Fusion Sci. Technol. 50, 327 (2006).

[3] T. Shimozuma et al., Nucl. Fusion 45, 1396 (2005).

[4] H. Takahashi et al., IAEA Fusion Energy Conference 2010, pp. EXC/P8, Daejon, Korea (2010).

[5] T. Shimozuma et al., J. Phys.: Conf. Series 123, 012022 (2008).

[6] M. Yokoyama et al., Nucl. Fusion 47, 1213 (2007).

[7] S. Matsuoka et al., Phys. Plasmas 18, 032511 (2011).

[8] A.H. Boozer, Phys. Fluids 24, 1999 (1981).

[9] S. Brunner et al., Phys. Plasmas 6, 4504 (1999).

[10] W.X. Wang et al., J. Plasma Fusion Res. SERIES 2, 250 (1999).

[11] S. Satake et al., Plasma Fusion Res. 3, S1062 (2008).

[12] W.I. van Rij and S.P. Hirshman, Phys. Fluids B 1, 563 (1989).

[13] A. Wakasa et al., Contrib. Plasma Phys. 50, 582 (2010).

[14] S. Matsuoka et al., Plasma Fusion Res. 6, 1203016 (2011).

[15] S.P. Hirshman and O. Betancourt, J. Comput. Phys. 96, 99 (1991).

[16] H. Sugama et al., Plasma Phys. Control. Fusion 53, 024004 (2011).

[17] K.H. Burrell, Phys. Plasmas 4, 1499 (1997).

[18] A. Fujisawa et al., Plasma Phys. Control. Fusion 48, S205 (2006).

[19] M. Nunami et al., Plasma Fusion Res. 5, 016 (2010). 